\title{
Clinical accuracy of non-contact infrared thermometer from umbilical region in children: A new side
}

\author{
Hurşit Apa ${ }^{1}$, Salih Gözmen², Şükran Keskin-Gözmen³, Fatma Aslan, Nuri Bayram, \\ İlker Devrim ${ }^{4}$ \\ Divisions of ${ }^{2}$ Pediatric Hematology, ${ }^{3}$ Pediatric Nephrology, and ${ }^{4}$ Pediatric Infectious Diseases, ${ }^{1}$ Department of Pediatrics, \\ Dr. Behcet Uz Children's Training and Research Hospital, Izmir, Turkey. E-mail: hur.apa@hotmail.com \\ Received: 28 August 2015, Revised: 7 January 2016, Accepted: 14 July 2016
}

SUMMARY: Apa H, Gözmen S, Keskin-Gözmen Ş, Aslan F, Bayram N, Devrim I. Clinical accuracy of non-contact infrared thermometer from umbilical region in children: A new side. Turk J Pediatr 2016; 58: 180-186.

Measurement from axillary site with digital thermometer has been accepted as the most accurate method. But this method is time consuming. Tympanic and forehead measurements are often used but don't always seem to be more appropriate. Another site, umbilical region, could be an alternative site. This study aims to compare the measurements with axillary digital thermometer and non-contact infrared thermometers at sites from umbilicus and forehead to determine whether umbilical site could be used accurately in children. For each method, 2,048 measurements in total were performed. Using axillary method as gold standard, with a cut-off temperature of $38^{\circ} \mathrm{C}$, the sensitivities and specificities, positive and negative predictive values of umbilical and forehead temperatures and area under the ROC curve were determined in non obese children. There was a significant positive correlation between axillary and umbilical temperatures with a correlation coefficient of 0.78 . The average difference between the mean of both axillary and umbilical temperatures was $-0.47 \pm 0.65^{\circ} \mathrm{C}$. The Bland-Altman plot showed good accuracy with only $2.5 \%$ of the readings falling outside the $95 \%$ level of confidence. Umbilical measurements showed sensitivity of $71.7 \%$ and specificity of $95.8 \%$. The area under the ROC curve was 0.93 .

The easy application may lead noncontact measurements from umbilicus site to be the preferable method for health care providers, but agreement limits mentioned in this study should be considered.

Key words: umbilical region, non-contact infrared thermometer, children.

Fever is a common chief complaint in the pediatric emergency room. Studies suggest that the height of fever, in combination with other clinical features, is a reliable predictor of occult bacteremia ${ }^{1-3}$. There are many methods and sites for measuring temperature. Each method and sites have their own advantages and disadvantages ${ }^{4-7}$. Oral and rectal temperatures are the most reliable predictors of core temperature ${ }^{8,9}$. Rectal measurement is assumed to be the clinical standard ${ }^{10,11}$. But measuring fever with oral or rectal route would sometimes be difficult because they are stressful for infants, are time-dependent and require acertain level of practice. These characteristics make them undesirable procedures for infants, health workers and parents ${ }^{1,12,13}$. The use of mercuryin-glass thermometers was banned in Turkey in 2010 according to the European Union legislation due to the toxicity of mercury. So, alternative devices and methods measuring from different sites-axillary, tympanic and forehead- had began to replace conventional device and methods in emergency rooms and hospitals. Measurements from axillary site with digital thermometers has been accepted as the most reliable and accurate method. But this method is time consuming, requiring special attention for 3 to 5 minutes to have a satisfactory measurement. So a non-contact, quick, hygienic, simple, accurate and inexpensive method would be more appropriate 
in pediatric practice ${ }^{14}$. Tympanic and forehead measurements often but not always seem to be more appropriate and easy to apply because sick children with high fever are generally agitated and do not do what caregiver want (e.g. measuring the temperature in an appropriate way) when it is most necessary and urgent. Tympanic measurements require the device to fit in the outer ear canal to have a satisfactory measurement. Measurement from forehead is quick, but sometimes it would be impossible to have a correct measurement when a child does not fix his head in front of the device approaching towards him for a few seconds during measurement. So, another site - umbilical- could be an alternative site to correctly measure the temperature of a child agitated because of illness, fever and crowded emergency room in a way that gives less discomfort. In pediatric age group, there are limited studies about non-contact infrared thermometers measuring from umbilical site.

This study aims to compare the measurements of body temperature with axillary digital thermometer and non-contact infrared thermometers at sites from umbilicus and forehead. The second aim is to determine whether non-contact method measuring temperature from umbilical site could be used accurately and interchangeably or instead of axillary digital measurements in children.

\section{Material and Methods}

Randomly selected, one hundred twenty two pediatric patients who were hospitalized in $\mathrm{Dr}$ Behcet Uz Children's Training and Research Hospital, Pediatric Infectious Disease Unit during March 2012 and October 2012 were included in the study. The height and weight of the patients were noted. Weight for age for patients under 2 years of age and body mass index (BMI) for patients older than 2 years of age were calculated. National survey data of growth charts for Turkish children published by Neyzi et al. ${ }^{15}$ was used to assess growth and related calculations. Patients who were $>95$ th percentile in weight for age under 2 years of age and $>25$ in BMI values for age for patients older than 2 years of age as well as patients with unstable conditions, including septic shock or circulatory collapse, patients with chronic diseases, including renal or liver failure, patients with ascites ${ }^{15}$, and patients with congenital or acquired abdominal anomalies (umbilical hernias, urachus remnant) were excluded from the study.

Written informed consent was obtained from the parents of the children. This study was approved by the local research ethics committee (2012/03-05, 26.04.2012). All the measurements were performed by the same nurse who was trained for each instrument.

Each patient was placed in a temperature controlled room between 24 and $26^{\circ} \mathrm{C}$ for 10 minutes before measurements were taken. Body temperature measurements were performed from axillary fossae with an axillary digital thermometer (Microlife MT 3001, Microlife AG Swiss Corporation, Widnau/ Switzerland) and from mid-forehead and from the area 1.5 $\mathrm{cm}$ below the umbilicus with a non-contact thermometer (ThermoFlash LX-26, Visiomed SAS France, Paris/France) at the same time. For each method, 2048 measurements in total were performed. Axillary temperature $\geq 38.0^{\circ} \mathrm{C}$ with digital thermometer was considered as fever ${ }^{8}$. All the temperatures were measured on the Celsius $\left({ }^{\circ} \mathrm{C}\right)$ scale. Before use, each thermometer was checked individually and compared with a standard thermometer, thus ensuring standardization and quality control with an accuracy of $\pm 0.1^{\circ} \mathrm{C}$. Noncontact thermometers were calibrated at the beginning of each study day. Axillary thermometers were calibrated at three months intervals in a water bath by placing the probe in the water for 10 min at a temperature of $38.0^{\circ} \mathrm{C}$. To ensure accurate results, the axillary region was dried using a towel before the measurements. The axillary thermometer was left in place until it electronically indicated that the appropriate time interval had passed. Noncontact thermometer was first calibrated to the room temperature and then held perpendicular to the area 1.5 $\mathrm{cm}$ below the umbilicus after 10 minutes of being undressed and to the mid-forehead. The operator held the device until a reliable reading had been taken.

Statistical analysis was done by using the Statistical Package for Social Sciences (SPSS) for Windows (Inc. Chicago USA, 2001) and Medcalc v.12.3.0 (MedCalc Software bvba, Broekstraat 52, 9030 Mariakerke, Belgium). Paired $t$ test was used to compare the mean temperature 
readings. Correlation was determined by the Pearson correlation coefficient while the extent of agreement was assessed with the Bland-Altman plot (1986) ${ }^{16}$. The limit of agreement was defined as \pm 2 SDs of the differences ${ }^{16}$. Using axillary method as gold standard, with a cut-off temperature of $38^{\circ} \mathrm{C}$, the sensitivities, and specificities, positive and negative predictive values of umbilical and forehead temperatures were determined. Receiver Operating Curves (ROC) was drawn and the area under the curve (AUC) with 95\% CI was also calculated. The level of significance was taken to be $\mathrm{p}<0.05$.

\section{Results}

One hundred patients met the inclusion criteria. The mean age of all 100 patients who participated in this study was $56.3 \pm 50.2$ months (between 1 and 168 months). Of the 100 patients, $53(53 \%)$ were male while 47 (47\%) were females. With 100 patients that participated in this study, we performed 2,048 temperature readings for every method. Mean BMI for patients over 2 years of age $(n=40)$ was 16.4 2.5 (range: 11.4-24.2). The number of axillary temperature readings under $38^{\circ} \mathrm{C}$ was 1594 and the number of axillary temperature readings over or equal to $38^{\circ} \mathrm{C}$ were 454 .

Mean axillary, forehead and umbilical temperatures with minimum and maximum readings were shown in Table $\mathrm{I}$. The mean umbilical temperature was significantly higher than both the mean forehead and axillary temperature when compared with paired $t$ test $(\mathrm{p}=0.00$ and $\mathrm{p}=0.00)$.

There was a significant positive correlation between axillary and umbilical temperatures with a correlation coefficient of 0.78 (p $<0.05$; 95\% CI 0.76-0.79). When we used temperature readings above or equal to $38^{\circ} \mathrm{C}$ with axillary method for correlation, the correlation coefficient between axillary and umbilical method was found to be 0.55 (p

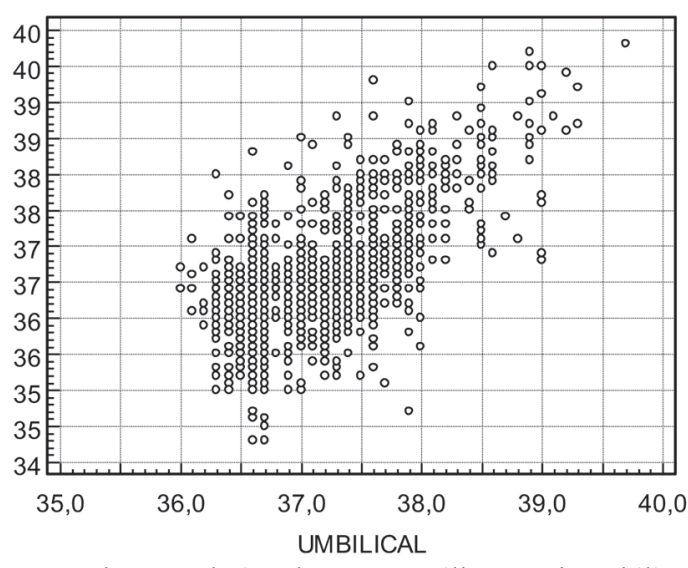

Fig. 1a. The correlation between axillary and umbilical temperature readings $(r=0.78, p<0.05,95 \%$ CI 0.76 to 0.79$)$.

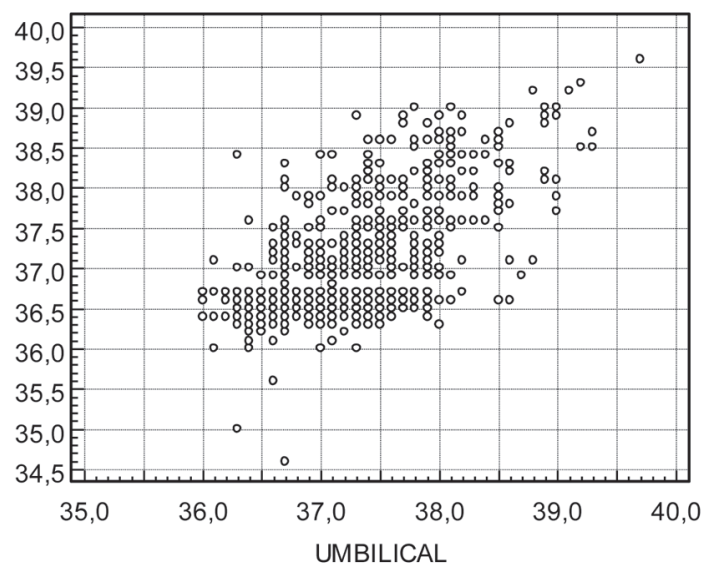

Fig. 1b. The correlation between forehead and umbilical temperature readings $(r=0.81 ; p<0.05,95 \%$ CI 0.79 to 0.82 )

$<0.05$; 95\% CI 0.48-0.61) When we compared forehead and umbilical temperature readings, we also found a significant positive correlation between these readings $(r=0.81 ; \mathrm{p}<0.05$, 95\% CI 0.79-0.82). These correlations were also significant at the 0.01 level. Correlation graphics with three methods were shown in Figure $1 \mathrm{a}$ and $1 \mathrm{~b}$.

The average difference between the mean of

Table I. Mean Axillary, Forehead and Umbilical Temperatures $\left({ }^{\circ} \mathrm{C}\right)$ with Ranges

\begin{tabular}{lccc}
\hline & $\mathrm{n}$ & Mean $(\mathrm{SD})$ & Range \\
\hline Forehead & 2,048 & $37.1(0.79)$ & $34.6-39.6$ \\
Axillary & 2,048 & $36.8(1.03)$ & $34.3-39.8$ \\
Umbiliculus & 2,048 & $37.3(0.75)$ & $36.0-39.7$ \\
\hline
\end{tabular}


both axillary and umbilical temperatures was $-0.47 \pm 0.65^{\circ} \mathrm{C}(95 \% \mathrm{CI}-0.49$ to -0.44$)$. The Bland-Altman plot showed that most of the data points were tightly clustered around the zero line of the difference between the two temperature readings with only $2.5 \%$ of the readings $(n=51)$ falling outside the $95 \%$ level of confidence (Figure 2a). When we used only temperature readings above or equal to $38^{\circ} \mathrm{C}$ with axillary method $(n=453)$ in the BlandAltman plot, plot showed that most of the data points were tightly clustered around the zero line of the difference between the two temperature readings with only $1.3 \%$ of the readings $(n=6)$ falling outside the $95 \%$ level of confidence.

The average difference between the mean of both forehead and umbilical temperatures was $-0.15 \pm 0.48{ }^{\circ} \mathrm{C}(95 \% \mathrm{CI}-0.22$ to -0.02$)$. The Bland-Altman plot showed that most of the data points were tightly clustered around the zero line of the difference between the two temperature readings with only $3.1 \%$ of the readings falling outside the $95 \%$ level of confidence (Fig. 2b).

When we considered $38^{\circ} \mathrm{C}$ as axillary temperature cutoff, the comparison with umbilical measurements showed sensitivity of $71.7 \%$ (95\% CI 67.4\%-75.8\%) and specificity of $95.8 \%$ (95\% CI $94.7 \%-96.7 \%)$. The positive predictive value of the umbilical measurements was $86.2 \%$ (95\% CI $82.1 \%-89.7 \%$ ), whereas the negative predictive value was $90.7 \%$ (95\% CI $89.2 \%-92.0 \%)$. The area under the ROC curve was 0.93 , indicating good accuracy (Fig. 3).

Positive and negative likelihood ratios were also calculated for umbilical method, assuming the axillary method as the criterion standard. Positive likelihood ratio was 22.04 and negative likelihood ratio was 0.36 for the umbilical measurement. When calculating the ROC curve to determine the best threshold for axillary temperature of greater than $38.0^{\circ} \mathrm{C}$, for an umbilical temperature of $37.6^{\circ} \mathrm{C}$ the sensitivity was $85.9 \%$, and the specificity was $89.8 \%$.

\section{Discussion}

The aim of temperature measurement is to obtain an accurate reflection of a patient's core temperature. As core temperature measurement requires invasive techniques, it is impractical to use them to achieve the criterion standard in routine practice ${ }^{6,17}$. The use of rectal mercury thermometers had long been the standard method for routine measurements but they were banned in Turkey in $2010^{13,17,18}$. So, alternative methods measuring from different sites-axillary, tympanic and forehead- began to replace conventional method in emergency rooms and inpatient services. But each method has its own advantages and disadvantages ${ }^{19}$. There is also disagreement about the optimal anatomic site for measuring body temperature ${ }^{13-15}$. Although infrared measurements from forehead is quick, sometimes it would be impossible to have a correct measurement especially when a child does not fix his head in front of the device for a sufficient amount of time to get a proper measurement in a crowded emergency room.

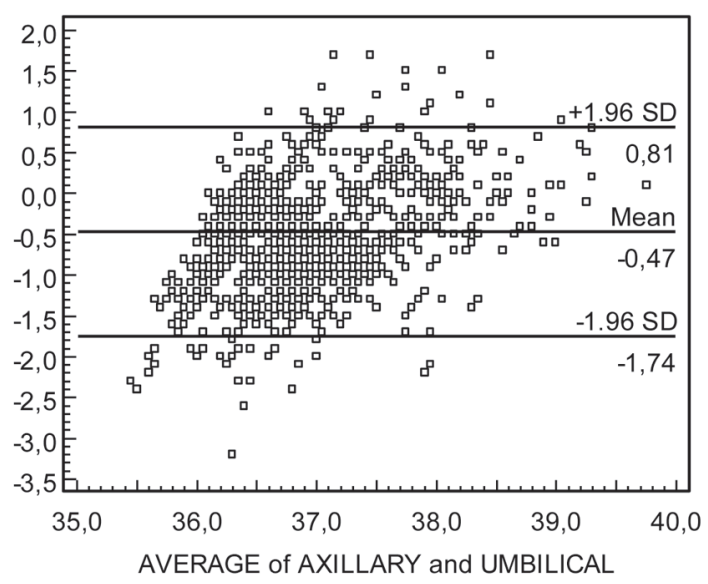

Fig. 2a. The Bland-Altman plot of difference comparing axillary and umbilical temperatures in the 2,048 readings, with mean difference and $95 \%$ limits of agreement.

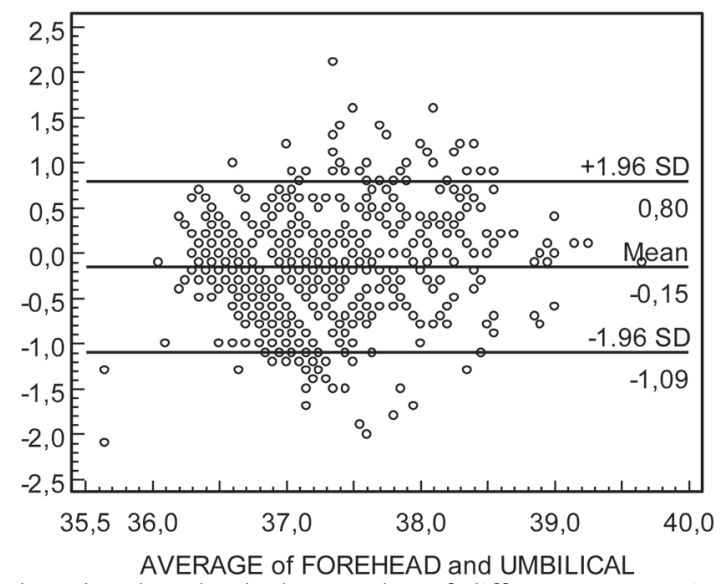

Fig. 2b. The Bland-Altman plot of difference comparing forehead and umbilical temperatures in the 2,048 readings, with mean difference and $95 \%$ limits of agreement. 


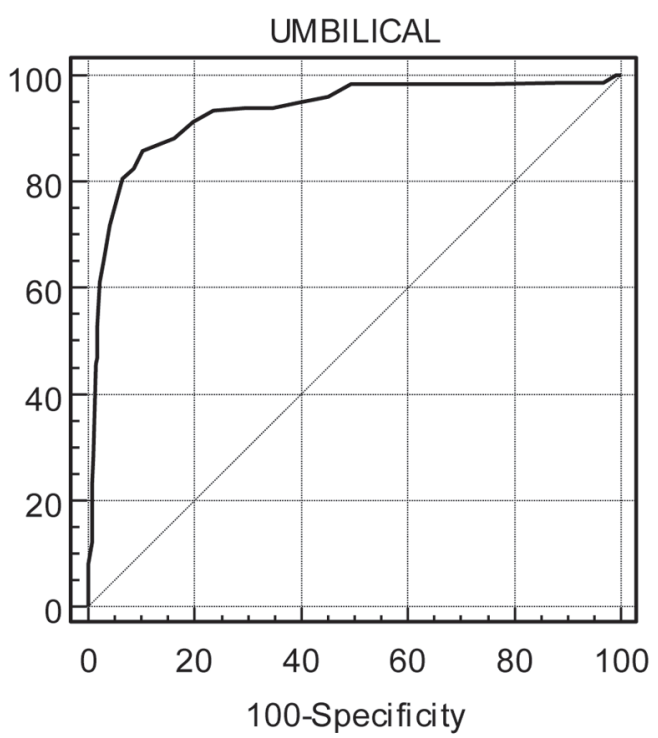

Fig. 3. Receiver operative characteristic curve for predicting febrile children with the umbilical method (axillary temperature $>38^{\circ} \mathrm{C}$ ). The area under the ROC curve is 0.93 (95\% CI, 0.92-0.94; $\mathrm{p}<0.05$ versus the identity line [diagonal line]).

These children with fever are generally agitated because of illness or fear of doctor or other health workers, crying and refuse any procedure that must be done for diagnosis. So, another site, the umbilical region could be an alternative site to get information about whether the child has a fever or not. In pediatric patient group, there are limited studies on non-contact infrared thermometers measuring from umbilical site. So, in this report, we aimed to demonstrate the body temperature measurements with infrared noncontact thermometers from umbilicus to determine whether this method and site can accurately replace axillary digital measurements as a sensitive, specific, and accurate method in children.

Before adopting new noninvasive methods of measurement, being quite certain that the new method will give results that agree with older methods and that can be subjected to recognize statistical procedures is important ${ }^{11}$. For this purpose, we performed four different approaches to compare three methods with each other. Firstly, we compared mean axillary, forehead and umbilical temperatures. Secondly, we performed correlations among them. Thirdly, we found the average difference between the means for each method and agreement with Bland-Altman plots. Finally, we calculated the specificity, sensitivity, as well as positive and negative predictive values of the umbilical method.

We found that the mean of umbilical measurements was significantly higher than the mean of forehead and axillary readings when compared with paired t test. But the mean temperature readings alone are not sufficiently valuable when deciding whether a method can be accurately used instead of another.

We found a significant positive correlation between axillary and umbilical measurements with a correlation coefficient of 0.78 as well as a significant positive correlation between forehead and umbilical temperature readings with $r=0.81$. In addition, there was a moderate positive correlation between axillary and umbilical measurements when we used only axillary readings above or equal to $38^{\circ} \mathrm{C}$. However, this high correlation alone does not always mean that these two methods can be accurately used instead of one another. Although numerous studies report correlations when comparing new devices to old; few examine whether the devices agree ${ }^{20}$.

The distribution of the temperature differences between a standard and the test method give a good indication of the accuracy of the method as expressed by Bland and Altman ${ }^{16}$. It should also be clearly established before the start of the investigation, what limits of deviation are acceptable. This point is overlooked in most cases. It is our opinion that before any new method of body temperature measurement is adopted, the method and instrumentation should guarantee that bias is low and 95\% of the results will be in limits of agreement.

We found the average difference (bias) between the mean of both axillary and umbilical temperatures as -0.47 , which seems very low with an SD of $0.65^{\circ} \mathrm{C}$. Bias is acceptable with value lower than $0.5^{\circ} \mathrm{C}$. Also, the Bland-Altman plot showed that most of the data points were tightly clustered around the zero line of the difference between two temperature readings. This also found to be true with axillary readings above or equal to $38^{\circ} \mathrm{C}$. The average difference between the mean of both forehead and umbilical temperatures was -0.15 with an SD of $0.48^{\circ} \mathrm{C}$. In Bland-Altman plot, $3.1 \%$ of the readings were falling outside the $95 \%$ level of CI. The distribution of the 
temperature differences between a standard and the test method give a good indication of the accuracy of the method as expressed by Bland and Altman. In our report, these results showed a good accuracy between umbilical and axillary/forehead methods.

When we considered $38^{\circ} \mathrm{C}$ as axillary temperature cutoff, the comparison with umbilical measurements showed sensitivity of $71.7 \%$ and specificity of $95.8 \%$. The positive predictive value of the umbilical measurements was $86.2 \%$, whereas the negative predictive value was $90.7 \%$. As the AUC was 0.93, the ROCs also supported these findings with greater accuracy.

A limitation of this study was that we did not have a true measure of the core body temperature to compare our temperature findings with. Core temperature refers to the measurement that most closely reflects the temperature in the blood flowing through the branches of the carotid arteries to the hypothalamus ${ }^{21}$. Because these sites are not routinely used in practice and the rectum measurements, which had been the criterion standard with mercury-in-glass thermometers, not being used anymore, we used axillary measurements using digital thermometer as the criterion standard. We excluded cases having values indicating overweight and obesity in our study as obesity has been associated with increased heat production as well as thick fat layer in the abdomen. Previous studies indicated that greater subcutaneous abdominal adipose tissue in obese cases provided a significant insulating layer blunting abdominal heat transfer ${ }^{22}$.

These results demonstrated that the umbilical method could be a good option in the measurement of fever in the pediatric population because it has high sensitivity, high specificity, and good agreement with a low bias. Having high positive likelihood ratio also makes this method valuable. The AUC makes this method reliable for the accurate definition of fever in the pediatric population. The easy application may lead noncontact measurements from umbilicus site to be the preferable method for health care providers, but agreement limits mentioned in this study should be considered.
1. Teran CG, Torrez-Llanos J, Teran-Miranda TE, et al. Clinical accuracy of a non-contact infrared skin thermometer in paediatric practice. Child Care Health Dev 2012; 38: 471-476.

2. Kara A, Devrim I, Cengiz AB, et al. Is the axilla the right site for temperature measurement in children by chemical thermometer? Turk J Pediatr 2009; 51: 325-327.

3. Mackowiak PA, Wasserman SS, Levine MM. A critical appraisal of 98.6 degrees $\mathrm{F}$, the upper limit of the normal body temperature, and other legacies of Carl Reinhold August Wunderlich. JAMA 1992; 268: 15781580.

4. Pocock G, Richards CD. Human Physiology The Basis of Medicine. New York, NY: Oxford University Press; 1999: 539-546.

5. Lorin M. Measurement of body temperature. Semin Pediatr Infect Dis 1993; 4: 4-8.

6. Fulbrook P. Core temperature measurements in adults: a literature review. J Adv Nurs 1993; 18: 1451-1460.

7. Apa H, Gozmen S, Bayram N, et al. Clinical accuracy of tympanic thermometer and noncontact infrared skin thermometer in pediatric practice. An alternative for axillary digital thermometer. Pediatr Emerg Care 2013; 29: 992-997.

8. Fischler MP, Reinhart WH. Fever: friend or enemy? Schweiz Med Wochenschr 1997; 127: 864-870.

9. Baraff LJ, Bass JW, Fleisher GR. Practice guideline for the management of infants and children 0 to 36 months of age with fever without source. Ann Emerg Med 1993; 22: 1198-1210.

10. Morley CJ, Hewson PH, Thornton AJ, et al. Axillary and rectal temperature measurements in infants. Arch Dis Child 1992; 67: 122-125.

11. Jensen BN, Jensen FS, Madsen SN, et al. Accuracy of digital tympanic, oral, axillary, and rectal thermometers compared with standard rectal mercury thermometers. Eur J Surg 2000; 166: 848-851.

12. Caravati EM, Erdman AR, Christianson G, et al Elemental mercury exposure: an evidence-based consensus guideline for out-of-hospital management. Clin Toxicol (Philia) 2008; 46: 1-21.

13. Schmitz T, Bair N, Falk M, et al. A comparison of five methods of temperature measurements in febrile intensive care patients. Am J Crit Care 1995; 4: 286 $-292$.

14. Chiappini E, Sollai S, Longhi R, et al. Performance of non-contact infrared thermometer for detecting febrile children in hospital and ambulatory settings. J Clin Nurs 2011; 20: 1311-1318.

15. Neyzi O, Günöz H, Furman A, Bundak R, Gökçay G, Darendeliler F, Baş F. Türk çocuklarında vücut ağırlığı, boy uzunluğu, baş çevresi ve vücut kitle indeksi referans değerleri. Çocuk Sağlığı ve Hastalıkları Dergisi 2008; 51: $1-14$

16. Bland JM, Altman DG. Statistical methods for assessing agreement between two methods of clinical measurement. Lancet 1986; 1: 307-310.

17. Cork RC, Vaughan RW, Humphrey LS. Precision and 
accuracy of intraoperative temperature monitoring. Anesth Analg 1983; 62: 211-214.

18. Jakobsson J, Nilsson A, Carlsson L. Core temperature measured in the auricular canal: comparison between four different tympanic thermometers. Acta Anaesthesiol Scand 1992; 36: 819-824.

19. Pocock G, Richards CD. Human Physiology The Basis of Medicine. New York, NY: Oxford University Press; 1999: 539-546.
20. Falzon A, Grech V, Caruana B, et al. How reliable is axillary temperature measurement? Acta Paediatr 2003; 92: 309-313.

21. Martin SA, Kline AM. Can there be a standard for temperature measurement in the pediatric intensive care unit? AACN Clin Issues 2004; 15: 254-266.

22. Savastano DM, Gorbach AM, Eden HS, Brady SM, Reynolds JC, Yanovski JA. Adiposity and human regional body temperature. Am J Clin Nutr 2009; 90: 1124-1131. 